\title{
Propensity-matched analysis of the efficacy of olanexidine gluconate versus chlorhexidine-alcohol as an antiseptic agent in thoracic esophagectomy
}

Takeo Fujita ${ }^{\text {* }}$, Naoya Okada' ${ }^{1}$ Takuji Sato ${ }^{1}$, Kazuma Sato ${ }^{1}$, Hisashi Fujiwara' ${ }^{1}$ Takashi Kojima ${ }^{2}$ and Hiroyuki Daiko ${ }^{3}$

\begin{abstract}
Background: In the present matched-cohort study, we investigated the efficacy of olanexidine gluconate in comparison with chlorhexidine-alcohol as an antiseptic agent in thoracic esophagectomy.

Methods: A total of 372 patients with esophageal cancer who were scheduled to undergo thoracic esophagectomy between 2016 and 2018 were assigned to one of two groups based on the preoperative antiseptic agent used in thoracic esophagectomy. We investigated the incidence of surgical site infectious complications in the propensitymatched cohort.

Results: Based on the propensity score, 116 patients prepared with 1.5\% olanexidine gluconate and 114 patients prepared with $1.0 \%$ chlorhexidine-alcohol as surgical skin antisepsis were selected. No significant intergroup differences were observed with respect to incisional surgical site infection $(0.8 \%$ in the olanexidine group versus $0.8 \%$ in the chlorhexidine group) and deep fascial/organ space surgical site infection (1.7\%/10.3\% in the olanexidine group versus $3.5 \% / 15.7 \%$ in the chlorhexidine group, $p=0.39 / p=0.03$ ). Notably, the respective incidences of surgical site infection except anastomotic leakage were $1.7 \%$ and $7.0 \%$ in the olanexidine and chlorhexidine groups $(p=0.04)$.

Conclusions: Olanexidine gluconate was well tolerated and significantly reduced incidence of surgical site infection except anastomotic leakage in comparison with chlorhexidine-alcohol as an antiseptic agent in thoracic esophagectomy with three-field lymph node dissection.
\end{abstract}

Keywords: Esophagectomy, Olanexidine gluconate, Chlorhexidine-alcohol, Propensity-matched analysis

\section{Background}

The use of optimal skin antiseptic agents is of critical importance, especially during traumatic surgical procedures $[1,2]$. One of the most common surgical complications is surgical site infection (SSI), particularly in gastrointestinal surgery. SSI prolongs the postoperative

\footnotetext{
*Correspondence: takfujit@east.ncc.go.jp

${ }^{1}$ Division of Esophageal Surgery, National Cancer Center Hospital East, Kashiwa, Japan

Full list of author information is available at the end of the article
}

hospital stay, which increases the medical cost. Multiple management protocols are followed to prevent the SSI. Of these, appropriate prophylactic antibiotics and skin antiseptic agents are the most important to minimize the risk of SSI. The major preoperative antiseptic agents that have been widely used as skin disinfectants throughout the world for a long time are chlorhexidine and povidone iodine [3, 4]. Recent clinical studies suggest that chlorhexidine-alcohol reduces the incidence of postoperative SSI compared with povidone iodine $[5,6]$. 
Olanexidine (Olanedine; Otsuka Pharmaceutical Factory, Inc., Tokushima, Japan), containing $1.5 \%$ olanexidine gluconate, is a new skin antiseptic agent that was approved in Japan in 2015. This newly developed antiseptic agent is a biguanide disinfectant with a similar structure to chlorhexidine, with a wide range of antimicrobial activity. Olanexidine is particularly effective against methicillin-resistant Staphylococcus aureus, vancomycin-resistant Enterococcus, Pseudomonas aeruginosa, and even Serratia marcescens compared with chlorhexidine $[7,8]$. Recent randomized control studies have demonstrated that olanexidine significantly reduces SSI compared with povidone iodine in clean-contaminated surgery $[5,9]$. However, it is unknown whether olanexidine is superior to chlorhexidine-alcohol in preventing SSI in clean-contaminated surgery. In the present study, we retrospectively investigated the incidence of SSI and performed a propensity matched-analysis to compare the efficacy of olanexidine gluconate versus chlorhexidinealcohol in clean-contaminated surgery, particularly in patients with esophageal cancer undergoing thoracic esophagectomy.

\section{Methods}

\section{Study design}

This study was a retrospective, single-center, two-arm cohort study performed between 2016 and 2018 at the National Cancer Center Hospital East, Japan. Eligible patients were assigned to receive skin disinfection with either $1.5 \%$ olanexidine gluconate (Olanedine ${ }^{\mathrm{TM}}$; Otsuka Yakuhin Kogyo, Tokyo, Japan) or $1.0 \%$ chlorhexidinealcohol (Sterichlone ${ }^{\mathrm{TM}}$; Kenei Seiyaku, Tokyo, Japan) $30 \mathrm{~min}$ before surgery, and 372 consecutive patients were analyzed. Written informed consent was obtained from all patients. The study was approved by the Committee for ethics of the National Cancer Center (Japan) (approval number \#2018-332). Also, this study confirms to the provisions of the Declaration of Helsinki (as revised in Tokyo 2004).

\section{Patients}

Patients who underwent thoracic esophagectomy for esophageal cancer at the National Cancer Center Hospital East, Japan, were investigated. Preoperative diagnoses were based on imaging studies, including upper gastrointestinal studies, endoscopy, and conventional crosssectional imaging (computed tomography). Histological evaluation of endoscope-guided biopsy specimens was performed in all cases. Data on the preoperative stage of the disease, histopathological findings, surgical procedures performed, and outcomes were collected from the patient medical records, as described previously [10].

In all cases, thoracic esophagectomy was performed under the direction of the regular attending surgeon.
For transthoracic esophagectomy, subtotal resection of the esophagus was performed with three-field regional lymph node dissection, regardless of the tumor stage. In the thoracoscopic approach, we preserved the azygos arch and the right bronchial artery. In contrast, the azygos arch was transected in all patients who underwent the thoracotomy approach. The laparoscopic approach was principally used, except in cases with bulky lymph node metastasis or previous history of abdominal surgery via laparotomy. The esophagus was usually reconstructed with a gastric tube via the retrosternal route; right hemicolic reconstruction was performed via the posterior sternal route when gastrectomy of the remnant stomach was required, as described previously [10].

\section{Anesthesia and intraoperative management during thoracic esophagectomy}

The standard institutional anesthetic practice for thoracic esophagectomy was modified to enable the development of intraoperative core hypothermia in the present study. In brief, upon patient arrival in the operating room, routine monitoring was applied, which included electrocardiography, noninvasive blood pressure monitoring, pulse oximetry, and capnography. Before the induction of anesthesia, an epidural catheter was inserted at the fifth to sixth thoracic interspace, and placed $5 \mathrm{~cm}$ beyond the introducing needle tip, as described previously [10].

Anesthesia was induced with $1.5-2.5 \mathrm{mg} / \mathrm{kg}$ propofol, $1-2 \mu \mathrm{g} / \mathrm{kg}$ fentanyl, and $0.1 \mathrm{mg} / \mathrm{kg}$ vecuronium. Anesthesia was maintained with $3 \%$ end-tidal sevoflurane in oxygen until tracheal intubation. Anesthesia was then maintained with $2 \%$ end-tidal sevoflurane at $40 \%$ oxygen (air/oxygen mixture at $4 \mathrm{~L} / \mathrm{min}$ ) supplemented with doses of fentanyl and vecuronium. A heat- and moisture-exchanging filter was positioned between the endotracheal tube and the breathing circuit, as described previously [10].

\section{Definition of surgical complications}

SSI was judged in accordance with the definition established by the Surgical Wound Infection Task Force 1, and included infections at the incision site or organ/space manipulated during operative intervention. Remote infections were not included in the definition of an SSI, with the exception of bloodstream infections related to a SSI. Symptomatic remote infections were also included in the analysis. Among the remote infections, respiratory infection was defined as the presence of new or progressive infiltrates on chest radiographs, plus at least two of the following signs of respiratory tract infection: temperature $>38^{\circ} \mathrm{C}$, purulent sputum, leukocytosis of $>1 \times 10^{4}$ / $\mathrm{mm}^{3}$ or leukopenia of $<4 \times 10^{3} / \mathrm{mm}^{3}$, and signs of 
inflammation on auscultation, as described previously [10].

\section{Perioperative management}

Perioperative management was performed with the same clinical management pathway (CMP) for all patients, regardless of the type of abdominal approach, as described previously [10]. All patients received enteral nutrition through a nasal feeding tube until the start of oral intake on postoperative day (POD) 6. In brief, fluid balance was achieved through a peripheral line, with additional enteral feeding on POD 1. Enteral nutrition was discontinued after the absence of anastomotic leakage was confirmed on POD 6. Perioperative antimicrobials are used only the surgery Cefmetazole sodium.

Perioperative management was performed by the same clinical staff in the same environment using the ICU and subsequent ward-based facilities. The same principles of care were applied to both groups. The CMP was applied in both the ICU (POD 1 and 2) and the surgical ward (POD 3 and later). In brief, the endotracheal tube was removed from all patients in the operating room or immediately upon arrival in the ICU. Patients remained in the ICU for 1 day after surgery. On POD 6, a radiographic contrast agent swallow examination was performed to evaluate the anastomosis and any passage problems. If this examination showed no leakage or obstruction, the nasogastric tube was removed and oral intake was initiated in accordance with the postoperative diet program. In the absence of any complications, the patient was enrolled in the postoperative rehabilitation program and discharged on POD $12-20$, as described previously [10].

If there were any abnormal clinical findings such as hypoxia, leukocytosis, or abnormal pleural drainage during the course of the postoperative CMP, computed tomography and radiographic examinations were performed to diagnose and optimally manage the abnormality as soon as possible as described previously [10].

\section{Statistical analysis}

Propensity score matching was used to assemble two comparable groups. The covariates were preoperative treatment, thoracic surgical approach, abdominal surgical approach, clinical stage, ASA Grade, and type of antibiotics. Each patient in the olanexidine gluconate group was matched to a patient in the chlorhexidine-alcohol group who had the closest propensity score using simple 1:1 matching without replacement. To prevent poor matches, a caliper of 0.20 of the standard deviation of the logit of the propensity score was used. Intergroup differences were analyzed using the Chi-squared test and the Mann-Whitney U-test. A $p$ value $<0.05$ was considered to indicate a statistically significant difference. All statistical analysis were performed using $\mathrm{R}$ calculation software.

\section{Results}

Of the 372 included patients, the antiseptic agent was chlorhexidine-alcohol in 178 patients, and 1.5\% olanexidine gluconate in 194 patients. Olanexidine gluconate was well tolerated and no adverse events specific to its administration were observed. All patients were successfully treated using the CMP without any problems. There were no significant differences between the chlorhexidine-alcohol and olanexidine gluconate groups with respect to baseline patient characteristics, including age, sex, body mass index, ASA grade, preoperative treatment, and clinical stage of the disease before adjusting (Table 1). Operative procedures and variables during esophagectomy in all 372 patients are shown in Table 2. There were no significant differences between groups in the thoracic and abdominal surgical approaches, total operative duration, total amount of intraoperative blood loss before adjusting (Table 2). There were no significant intergroup differences regarding the immediate pre- and postoperative skin reaction before and after esophagectomy (data not shown). A summary of the short-term outcomes after esophagectomy in all 372 patients before adjusting are presented in Table 3. There were no significant differences between groups in the 30-day incidence of SSI $(16.4 \%$ in the chlorhexidine-alcohol group versus $17.4 \%$ in the olanexidine gluconate group, $p=0.81)$, incidence of anastomotic leakage $(12.3 \%$ in the

Table 1 Patients' characteristics

\begin{tabular}{|c|c|c|c|}
\hline Variables & $\begin{array}{l}\text { Olanexidine group } \\
(n=194)\end{array}$ & $\begin{array}{l}\text { Chlorhexidine } \\
\text { group } \\
(n=178)\end{array}$ & p value \\
\hline Age (mean \pm S.D.) & $68.5 \pm 4.4$ & $67.6 \pm 5.9$ & 0.21 \\
\hline Gender (M:F) & $158: 36$ & $151: 27$ & 0.38 \\
\hline $\begin{array}{l}\text { Body Mass Index } \\
(\text { mean } \pm \text { S.D.) }\end{array}$ & $21.5 \pm 2.6$ & $21.7 \pm 2.8$ & 0.55 \\
\hline ASA Grade & & & 0.39 \\
\hline Grade 1 & 78 & 64 & \\
\hline Grade 2 & 116 & 114 & \\
\hline $\begin{array}{l}\text { Pre-operative treat- } \\
\text { ment }\end{array}$ & & & 0.43 \\
\hline Chemotherapy & 95 & 92 & \\
\hline Chemo-radiation & 17 & 12 & \\
\hline $\begin{array}{l}\text { Clinical stage (UICC } \\
\text { 7th) }\end{array}$ & & & 0.65 \\
\hline Stage I & 47 & 51 & \\
\hline Stage II & 49 & 48 & \\
\hline Stage III & 77 & 68 & \\
\hline Stage IV & 17 & 11 & \\
\hline
\end{tabular}


Table 2 Operative procedure and variables during esophagectomy

\begin{tabular}{llll}
\hline Variables & $\begin{array}{l}\text { Olanexidine } \\
\text { group } \\
\text { (n= 194) }\end{array}$ & $\begin{array}{l}\text { Chlorhexidine } \\
\text { group } \\
\text { (n=178) }\end{array}$ & p value \\
\hline $\begin{array}{l}\text { Type of surgical approach } \\
\quad \text { Thoracoscopic }\end{array}$ & 161 & 153 & 0.43 \\
$\quad$ Thoracotomy & 33 & 25 & \\
$\begin{array}{l}\text { Type of surgical approach } \\
\text { Laparoscopic }\end{array}$ & 165 & 157 & 0.37 \\
$\quad$ Laparotomy & 29 & 21 & \\
$\begin{array}{l}\text { Total time of procedure (min) } \\
\text { Total amount of blood loss }\end{array}$ & 383.6 & 368.1 & 0.22 \\
during surgery (ml) & 183.2 & 211.5 & 0.44 \\
\hline
\end{tabular}

Table 3 Postoperative events and success rate of the CMP

\begin{tabular}{|c|c|c|c|}
\hline Variables & $\begin{array}{l}\text { Olanexidine group } \\
(\mathrm{n}=194)\end{array}$ & $\begin{array}{l}\text { Chlorhexidine } \\
\text { group } \\
(n=178)\end{array}$ & $\mathrm{p}$ value \\
\hline 30-days SSI (\%) & $32(16.4)$ & $31(17.4)$ & 0.81 \\
\hline $\begin{array}{l}\text { Anastomotic leakage } \\
(\%)\end{array}$ & $24(12.3)$ & $22(12.3)$ & 0.99 \\
\hline In hospital death (\%) & $1(0.7)$ & $1(0.7)$ & 0.95 \\
\hline $\begin{array}{l}\text { Success rate of CMP } \\
(\%)\end{array}$ & $140(72.1)$ & $139(78.0)$ & 0.18 \\
\hline
\end{tabular}

chlorhexidine-alcohol group versus $12.3 \%$ in the olanexidine gluconate group, $p=0.99$ ), or in-hospital deaths $(p=0.95)$. The success rates of the CMP were similar in both groups $(72.1 \%$ in the chlorhexidine-alcohol group versus $78.0 \%$ in the olanexidine gluconate group; $p=0.18$ ).

The patients' variables after propensity score matching are shown in Table 4. There were no significant differences between groups in age, sex, body mass index, ASA grade, preoperative treatment, clinical stage of the disease, and type of surgical approaches. Postoperative events and surgical outcomes are shown in Table 5. There were no significant differences between the matched groups in the total incidence of SSI $(12.0 \%$ in the chlorhexidine-alcohol group versus $20.1 \%$ in the olanexidine gluconate group, $p=0.09$ ), incidence of incisional or deep SSI (incisional SSI: $0.8 \%$ in the chlorhexidinealcohol group versus $0.8 \%$ in the olanexidine gluconate group, $p=0.99$; deep fascial SSI: $1.7 \%$ in the chlorhexidine-alcohol group versus $3.5 \%$ in the olanexidine gluconate group, $p=0.39$; organ space SSI: $10.3 \%$ in the chlorhexidine-alcohol group versus $15.7 \%$ in the olanexidine gluconate group, $p=0.30$; organ space SSI without anastomotic leakage: $0 \%$ in the chlorhexidine-alcohol
Table 4 Patients' characteristics in the propensity-matched cohort

\begin{tabular}{|c|c|c|c|}
\hline Variables & $\begin{array}{l}\text { Olanexidine group } \\
(n=116)\end{array}$ & $\begin{array}{l}\text { Chlorhexidine } \\
\text { group } \\
(n=114)\end{array}$ & $\mathrm{p}$ value \\
\hline Age (mean \pm S.D.) & $67.9 \pm 3.7$ & $67.8 \pm 4.4$ & 0.51 \\
\hline Gender (M:F) & 103:13 & $101: 13$ & 0.96 \\
\hline $\begin{array}{l}\text { Body Mass Index } \\
\text { (mean } \pm \text { S.D.) }\end{array}$ & $22.1 \pm 2.2$ & $21.9 \pm 2.3$ & 0.61 \\
\hline ASA Grade & & & 0.47 \\
\hline Grade 1 & 45 & 39 & \\
\hline Grade 2 & 71 & 75 & \\
\hline $\begin{array}{l}\text { Pre-operative treat- } \\
\text { ment }\end{array}$ & & & 0.13 \\
\hline Chemotherapy & 71 & 47 & \\
\hline Chemo-radiation & 8 & 11 & \\
\hline $\begin{array}{l}\text { Clinical stage (UICC } \\
\text { 7th) }\end{array}$ & & & 0.99 \\
\hline Stage I & 29 & 30 & \\
\hline Stage II & 20 & 22 & \\
\hline Stage III & 59 & 55 & \\
\hline Stage IV & 8 & 7 & \\
\hline $\begin{array}{l}\text { Type of surgical } \\
\text { approach }\end{array}$ & & & 0.47 \\
\hline Thoracoscopic & 98 & 100 & \\
\hline Thoracotomy & 18 & 14 & \\
\hline $\begin{array}{l}\text { Type of surgical } \\
\text { approach }\end{array}$ & & & 0.56 \\
\hline Laparoscopic & 101 & 103 & \\
\hline Laparotomy & 15 & 11 & \\
\hline
\end{tabular}

Table 5 Postoperative events and success rate of the CMP in the propensity-matched cohort

\begin{tabular}{|c|c|c|c|}
\hline Variables & $\begin{array}{l}\text { Olanexidine group } \\
(\mathrm{n}=116)\end{array}$ & $\begin{array}{l}\text { Chlorhexidine } \\
\text { group } \\
(n=114)\end{array}$ & p value \\
\hline 30-days SSI (\%) & $14(12.0)$ & $23(20.1)$ & 0.09 \\
\hline Incisional SSI (\%) & $1(0.8)$ & $1(0.8)$ & 0.99 \\
\hline Deep SSI (\%) & $2(1.7)$ & $4(3.5)$ & 0.39 \\
\hline Organ space SSI (\%) & $12(10.3)$ & $18(15.7)$ & 0.30 \\
\hline $\begin{array}{l}\text { Organ space SSI } \\
\text { without anasto- } \\
\text { motic leakage (\%) }\end{array}$ & $0(0)$ & $3(2.6)$ & 0.23 \\
\hline $\begin{array}{l}\text { Anastomotic leakage } \\
(\%)\end{array}$ & $12(10.3)$ & $15(13.1)$ & 0.50 \\
\hline $\begin{array}{l}\text { Postoperative hospital } \\
\text { stay (median } \pm \text { IQR) }\end{array}$ & $15.0 \pm 3.8$ & $15.0 \pm 4.2$ & 0.88 \\
\hline In hospital death (\%) & $0(0)$ & $0(0)$ & - \\
\hline $\begin{array}{l}\text { Success rate of CMP } \\
(\%)\end{array}$ & $84(72.4)$ & $85(74.5)$ & 0.71 \\
\hline
\end{tabular}


group versus $2.6 \%$ in the olanexidine gluconate group, $p=0.23)$. There were no difference in median postoperative hospital stay $(\mathrm{p}=0.88)$. There were no in-hospital deaths in either group, and the success rates of the CMPs were similar in both groups $(72.4 \%$ in the chlorhexidinealcohol group versus $74.5 \%$ in the olanexidine gluconate group; $p=0.71$ ). Notably, there was significant difference between groups in the incidence of all SSI with no association with anastomotic leakage $(1.7 \%$ in the chlorhexidine-alcohol group versus $7.0 \%$ in the olanexidine gluconate group, $p=0.04$ ) (Fig. 1).

\section{Discussion}

After the development of anesthetic techniques, the surgical field was poised to expand the number of operative procedures that could be performed safely. Among the perioperative surgical management factors, optimization of skin antiseptic agents is considered to be of great importance in reducing SSI. Povidone iodine and chlorhexidine have been widely used as skin antiseptic agents in various types of surgery for many years. The efficacy of these agents was investigated extensively in many types of procedures, including surgery and catheter-associated medical procedures [11]. Several clinical studies suggest that chlorhexidine-alcohol reduces the incidence of SSI in comparison with povidone iodine in clean-contaminated surgery $[5,12]$. However, although numerous clinical studies have examined which antiseptic skin agent is more effective in preventing SSI, there is still no robust consensus [13].

Olanexidine gluconate is a new antiseptic skin agent developed and approved in Japan. Olanexidine gluconate has antimicrobial activity against a wide range of bacterial species, including Gram-positive and Gram-negative bacteria [7, 14]. Furthermore, olanexidine gluconate reportedly has higher antimicrobial activity against drugresistant species (such as methicillin-resistant $S$. aureus and vancomycin-resistant Enterococci) than other antiseptic skin agents, including chlorhexidine-alcohol or povidone iodine $[7,8]$. Several clinical trials have shown the efficacy of olanexidine gluconate in various types of surgery [15-17]. A recent randomized control study reported that the 30-day incidence of SSI in various types of clean-contaminated surgery was $7 \%$ in the olanexidine group and $13.3 \%$ in the povidone iodine group (adjusted risk reduction: $0.48,90 \%$ CI $0.30-0.74 ; p=0.002$ ), while there were no significant intergroup differences in the incidence of deep incisional SSI (adjusted risk reduction: $0.55,90 \%$ CI $0.29-1.03 ; p=0.06$ ) [18]; therefore, the authors concluded that olanexidine gluconate is significantly associated with a low incidence of superficial SSI. However, to our knowledge, no study has investigated the efficacy of olanexidine gluconate in comparison with chlorhexidine-alcohol in clean-contaminated surgery.

It is widely recognized that there are several types of surgical procedures that have accompanying relatively high incidences of SSI in digestive surgery. Patients who undergo thoracic esophagectomy have a higher risk of SSI than those who undergo other types of digestive surgery [19, 20]. In the present study, using a propensity-matched cohort, we compared the incidence of SSI after thoracic esophagectomy with olanexidine gluconate or chlorhexidine-alcohol as the antiseptic skin agent. Although there was no significant

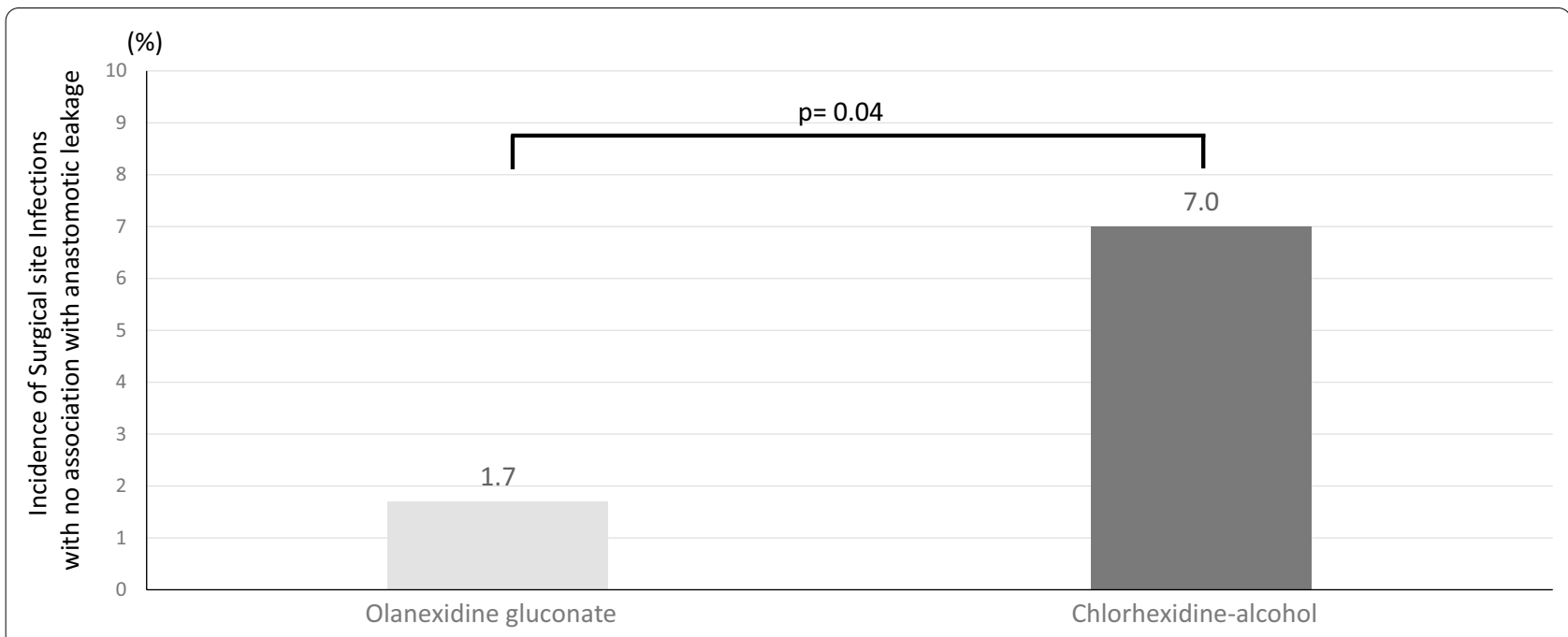

Fig. 1 Incidence of surgical site infection (SSI) without anastomotic leakage. Although there were no significant differences between groups in the incidence of SSI without anastomotic leakage, patients prepared with olanexidine gluconate had a lower incidence of SSI 
intergroup difference in the overall incidence of SSI, the incidence of SSI tended to be lower in patients prepared with olanexidine gluconate $(12.0 \%)$ than those prepared with chlorhexidine-alcohol (20.1\%). It is widely recognized that anastomotic leakage in thoracic esophagectomy is associated with multiple factors, such as patient nutrition and anatomical factors, which are usually unrelated to the skin preparation with antiseptic agents. Therefore, we further investigated the incidence of SSI without anastomotic leakage. Notably, olanexidine gluconate significant reduced the incidence of SSI with no association with anastomotic leakage in comparison with chlorhexidine-alcohol. However, there were no difference in the postoperative hospital stay. Since esophageal cancer surgery is highly invasive, the length of hospital stay for SSI management is not expected to have a significant overall impact in hospital stay unless it is caused by anastomotic leakage. The results were consistent with the overall incidence of SSI in that patients prepared with olanexidine gluconate tended to have a lower incidence of SSI without anastomotic leakage than those prepared with chlorhexidine-alcohol. We could not clearly describe the reason of the superiority of olanexidine gluconate. However, the results of previous clinical studies suggest the superiority of olanexidine gluconate, which is consistent with the results of this study. In addition, previous results of basic research has shown that olanexidine gluconate has high biological activity, especially against drug-resistant bacteria, which may help to interpret the results of this study.

The present study has several limitations. First, this study was investigated retrospectively. A recent randomized control study revealed that olanexidine gluconate significantly reduces the occurrence of overall SSI and superficial incisional SSI compared with povidone iodine in clean-contaminated surgery [18]. Therefore, further study is required to fully reveal the superiority of olanexidine gluconate in comparison with chlorhexidinealcohol. Second, this study covered a period of more than 2 years, during which time the thoracic and abdominal surgical devices differed slightly. However, the anesthesia and perioperative patient management procedures were performed consistently with the same CMP, which may be considered a strength of the study. Additionally, the prevalence of preoperative treatment was slightly lower in the chlorhexidine-alcohol group than in the olanexidine group $(p=0.13)$. Usually, patients who undergo preoperative treatment have a lower white blood cell count, which potentially makes them more vulnerable to bacterial infections [21]. However, the white blood cell count before surgery did not significantly differ between groups (data not shown). Furthermore, even though more patients were treated preoperatively in the olanexidine gluconate group than the chlorhexidine-alcohol group, the incidences of SSI were not increased.

\section{Conclusions}

In conclusion, present study firstly report the direct comparison between olanexidine gluconate and chlorhexidine-alcohol. The present results indicate that olanexidine gluconate as an antiseptic agent is effective for the prevention of surgical site infectious complications after thoracic esophagectomy with three-field lymph node dissection. Further analysis of a larger number of cases in a randomized controlled study would provide detailed results on the potential influence of olanexidine gluconate in thoracic esophagectomy.

\section{Abbreviations}

SSI: Surgical site infection; POD: Post-operative day; CMP: Clinical management pathway.

\section{Acknowledgements}

We greatly appreciate the members of the Division of Esophageal Surgery for their critical discussion of our manuscript. We thank the members of the Division of Anesthesiology for reviewing and discussing the study. We also thank Kelly Zammit, BVSc, from Edanz Group (https://en-author-services.edanz.com/ ac) for editing a draft of this manuscript.

\section{Author contributions}

TF, ON, TS, KS and HF made substantial contributions to the conception and design, acquisition of data, and analysis and interpretation of data. TF drafted the manuscript, and TK and HD substantively reviewed and revised it. All persons who meet authorship criteria are listed as authors, and all authors certify that they have participated sufficiently in the work to take public responsibility for the content, including participation in the concept, design, analysis, writing, or revision of the manuscript. All authors read and approved the final manuscript.

Funding

Not applicable.

\section{Availability of data and materials}

The datasets used and/or analysis during the current study are available from the corresponding author on reasonable request.

\section{Declarations}

Ethics approval and consent to participate

The study protocol was approved by the Committee foe Ethics of National Cancer Center (Japan) (Approval Number \#2018-332). The requirement of informed consent was waived due to the retrospective study design.

\section{Consent for publication}

Not applicable.

\section{Competing interests}

There are no competing interests to declare.

\section{Author details}

${ }^{1}$ Division of Esophageal Surgery, National Cancer Center Hospital East, Kashiwa, Japan. ${ }^{2}$ Division of Gastrointestinal Oncology, National Cancer Center Hospital East, Kashiwa, Japan. ${ }^{3}$ Division of Esophageal Surgery, National Cancer Center Hospital, Tokyo, Japan. 
Received: 8 May 2021 Accepted: 5 January 2022

Published online: 22 January 2022

\section{References}

1. Ceviker K, Canikoglu M, Tatlioglu S, et al. Reducing the pathogen burden and promoting healing with polyhexanide in non-healing wounds: a prospective study. J Wound Care. 2015;24:582-6.

2. Mangram AJ, Horan TC, Pearson ML, et al. Guideline for prevention of surgical site infection, 1999. Centers for Disease Control and Prevention (CDC) hospital infection control practices advisory committee. Ann J Infect Control. 1999;1999(27):97-132.

3. Dumville JC, McFarlane E, Edwards P, et al. Preoperative skin antiseptics for preventing surgical wound infections after clean surgery. Cochrane Database Syst Rev. 2015. https://doi.org/10.1002/14651858.CD003949. pub4.

4. Spaziani E, Di Filippo A, Orelli S, et al. Pre-operative skin antisepsis with chlorhexidine gluconate and povidone-iodine to prevent port-site infection in laparoscopic cholecystectomy: a prospective study. Surg Infect (Larchmt). 2018;19:334-8.

5. Darouiche RO, Wall MJ Jr, Itani KM, et al. Chlorhexidine-alcohol versus povidone-iodine for surgical-site antisepsis. N Engl J Med. 2010:362:18-26

6. Ritter B, Herlyn PKE, Mittlmeier T, et al. Preoperative skin antisepsis using chlorhexidine may reduce surgical wound infections in lower limb trauma surgery when compared to povidone-iodine-a prospective randomized trial. Am J Infect Control. 2020:48:167-72.

7. Inoue Y, Hagi A, Nii T, et al. Novel antiseptic compound OPB-2045G shows potent bactericidal activity against methicillin-resistant Staphylococcus aureus and vancomycin-resistant Enterococcus both in vitro and in vivo: a pilot study in animals. J Med Microbiol. 2015;64:32-6

8. Hagi A, Iwata K, Nii T, et al. Bactericidal effects and mechanism of action of olanexidine gluconate, a new antiseptic. Antimicrob Agents Chemother. 2015:59:4551-9.

9. Noorani A, Rabey N, Walsh SR, et al. Systematic review and meta-analysis of preoperative antisepsis with chlorhexidine versus povidone-iodine in clean-contaminated surgery. Br J Surg. 2010;97:1614-20.

10. Fujita T, Okada N, Horikiri Y, et al. Safety and efficacy of hydroxyethyl starch 6\% 130/0.4/9 solution versus 5\% human serum albumin in thoracic esophagectomy with 3-field lymph nodes dissection. Surg Today. 2019:49:427-34

11. Fitzgerald L, Kurtz J, Knepper BC, et al. Prepping for prevention: an intervention to optimize skin antisepsis and peri-operative antibiotic prophylaxis. Surg Infect (Larchmt). 2020;21:48-53.

12. Srinivas A, Kaman L, Raj P, et al. Comparison of the efficacy of chlorhexidine gluconate versus povidone iodine as preoperative skin preparation for the prevention of surgical site infections in clean-contaminated upper abdominal surgeries. Surg Today. 2015;45:1378-84.

13. Chen S, Chen JW, Guo B, et al. Preoperative antisepsis with chlorhexidine versus povidone-iodine for the prevention of surgical site infection: a systematic review and meta-analysis. World J Surg. 2020;44:1412-24.

14. Nakata H, Tsubotani Y, Nii T, et al. Effects of olanexidine gluconate on preoperative skin preparation: an experimental study in cynomolgus monkeys. J Med Microbiol. 2017;66:678-85.

15. Asukai M, Ohishi T, Fujita T, et al. Olanexidine gluconate versus povidoneiodine for preventing surgical-site infection in orthopaedic surgery: a retrospective study. J Orthop Sci. 2019;24:1125-9.

16. Yamamoto M, Hara K, Sugezawa K, et al. Disinfection with single or double usage of new antiseptic olanexidine gluconate in general surgery: a randomized study. Langenbecks Arch Surg. 2020;405:1183-9.

17. Nakaminami $\mathrm{H}$, Takadama S, Okita M, et al. Fast-acting bactericidal activity of olanexidine gluconate against qacA/B-positive methicillin-resistant Staphylococcus aureus. J Med Microbiol. 2019;68:957-60.

18. Obara $\mathrm{H}$, Takeuchi $\mathrm{M}$, Kawakubo $\mathrm{H}$, et al. Aqueous olanexidine versus aqueous povidone-iodine for surgical skin antisepsis on the incidence of surgical site infections after clean-contaminated surgery: a multicentre, prospective, blinded-endpoint, randomised controlled trial. Lancet Infect Dis. 2020;20:1281-9.

19. Yin $L X$, Chen $B M$, Zhao GF, et al. Scoring system to predict the risk of surgical site infection in patients with esophageal cancer after esophagectomy with cervical anastomosis. Dis Esophagus. 2008;21:266-71.

20. Nobuhara $H$, Yanamoto $S$, Funahara $M$, et al. Effect of perioperative oral management on the prevention of surgical site infection after colorectal cancer surgery: a multicenter retrospective analysis of 698 patients via analysis of covariance using propensity score. Medicine (Boltimore). 2018:97:e12545.

21. Nederlof N, Slaman AE, van Hagen P, et al. Using the comprehensive complication index to assess the impact of neoadjuvant chemoradiotherapy on complication severity after esophagectomy for cancer. Ann Surg Oncol. 2016;23:3964-71.

\section{Publisher's Note}

Springer Nature remains neutral with regard to jurisdictional claims in published maps and institutional affiliations.
Ready to submit your research? Choose BMC and benefit from:

- fast, convenient online submission

- thorough peer review by experienced researchers in your field

- rapid publication on acceptance

- support for research data, including large and complex data types

- gold Open Access which fosters wider collaboration and increased citations

- maximum visibility for your research: over $100 \mathrm{M}$ website views per year

At BMC, research is always in progress.

Learn more biomedcentral.com/submissions 